\title{
AN INEQUALITY FOR POLYNOMIALS
}

\author{
M. A. Malik
}

(received August 17, 1962)

Throughout this note let $\mathrm{p}(\mathrm{z})=\sum_{\nu=0}^{\mathrm{n}} \mathrm{a}_{\nu} z^{\nu}$ be a polynomial of degree n. The following results a re immediate.

THEOREM A. For $\mathrm{R} \geqq 1$

(1) $\quad \int_{0}^{2 \pi}\left|p\left(R e^{i \theta}\right)\right|^{2} d \theta \leqq R^{2 n} \int_{0}^{2 \pi}\left|p\left(e^{i \theta}\right)\right|^{2} d \theta$

THEOREM B. If $\mathrm{p}(\mathrm{z})$ has no zeros in $|\mathrm{z}|<1$, then

(2) $\quad \int_{0}^{2 \pi}\left|p\left(R e^{i \theta}\right)\right|^{2} d \theta \leqq \frac{R^{2 n}+1}{2} \int_{0}^{2 \pi}\left|p\left(e^{i \theta}\right)\right|^{2} d \theta$

for $R \geqq 1$

Theorem B was proved by Q. I. Rahman [2]. This result is the best possible and equality holds for $\mathrm{p}(\mathrm{z})=\alpha+\beta \mathrm{z}^{\mathrm{n}}$ where $|\alpha|=|\beta|$.

We prove the following

THEOREM 1. If $\mathrm{p}(\mathrm{z})$ has no zeros in $|\mathrm{z}|<\mathrm{k}, \mathrm{k} \geqq 1$, then

(3) $\quad \int_{0}^{2 \pi}\left|p\left(\operatorname{Re}^{i \theta}\right)\right|^{2} d \theta \leqq \frac{R^{2 n}+k^{2 n}}{1+k^{2 n}} \int_{0}^{2 \pi}\left|p\left(e^{i \theta}\right)\right|^{2} d \theta$

Canad. Math. Bull. vol. 6, no.1, January 1963. 
$\underline{\text { for }} R \stackrel{k^{2}}{=}$ And

(4) $\quad \int_{0}^{2 \pi}\left|p\left(R e^{i \theta}\right)\right|^{2} d \theta \leqq R^{n} \int_{0}^{2 \pi}\left|p\left(e^{i \theta}\right)\right|^{2} d \theta$

for $1 \leqq R \leqq k^{2}$.

If $\mathrm{p}(\mathrm{z})=\alpha \mathrm{k}^{\mathrm{n}}+\beta \mathrm{z}^{\mathrm{n}}$, where $|\alpha|=|\beta|$, then (3) becomes an equality.

Proof of Theorem 1. To start with let us suppose that $p(z)$ has all its zeros on $|z|=k$, so that $p(z)=a \prod_{t=1}^{n}\left(z-z_{t}\right)$. where $\left|\mathrm{z}_{1}\right|=\left|\mathrm{z}_{2}\right|=\ldots=\left|\mathrm{z}_{\mathrm{n}}\right|=\mathrm{k}$. Hence if $\mathrm{p}(\mathrm{z})=\sum_{\nu=0}^{\mathrm{n}} \mathrm{a}_{\nu} \mathrm{z}^{v}$, then $\left|a_{v}\right|=k^{n-2 v}\left|a_{n-v}\right|$ and

$$
\begin{aligned}
\int_{0}^{2 \pi}\left|\mathrm{p}\left(\mathrm{Re}^{\mathrm{i} \theta}\right)\right|^{2} \mathrm{~d} \theta & =2 \pi \sum_{v=0}^{\mathrm{n}}\left|\mathrm{a}_{v}\right|^{2} \mathrm{R}^{2 v} \\
& =\pi \sum_{v=0}^{\mathrm{n}} \frac{\mathrm{R}^{2 \mathrm{n}-2 v}+\mathrm{k}^{2 \mathrm{n}-4 v} \mathrm{R}^{2 v}}{1+\mathrm{k}^{2 \mathrm{n}-4 v}} \\
& \times\left(\left|\mathrm{a}_{\mathrm{n}-v}\right|^{2}+\left|\mathrm{a}_{v}\right|^{2}\right) .
\end{aligned}
$$

Now, if $0 \leqq v \leqq n$ then

$$
\begin{aligned}
& \frac{R^{2 n}+k^{2 n}}{1+k^{2 n}}-\frac{k^{2 n-4 v} R^{2 v}+R^{2 n-2 v}}{1+k^{2 n-4 v}} \\
& =\frac{\left(R^{2 v}-k^{4 v}\right)\left(R^{2 n-2 v}-1\right) k^{2 n-4 v}+\left(R^{2 v}-1\right)\left(R^{2 n-2 v}-k^{4 n-}\right.}{\left(1+k^{2 n}\right)\left(1+k^{2 n-4 v}\right)} \\
& \geq 0
\end{aligned}
$$


for $R \stackrel{2}{=} k^{2}$. Thus the greatest of the quantities

$\frac{\mathrm{R}^{2 \mathrm{n}-2 v}+\mathrm{k}^{2 \mathrm{n}-4 v} \mathrm{R}^{2 v}}{1+\mathrm{k}^{2 \mathrm{n}-4 v}}$ for $v=0,1,2, \ldots, \mathrm{n}$ is $\frac{\mathrm{R}^{2 \mathrm{n}}+\mathrm{k}^{2 \mathrm{n}}}{1+\mathrm{k}^{2 \mathrm{n}}}$

if $\mathrm{R} \geq \mathrm{k}^{2}$, and

$$
\int_{0}^{2 \pi}\left|p\left(R e^{i \theta}\right)\right|^{2} d \theta \leqq \frac{R^{2 n}+k^{2 n}}{1+k^{2 n}} \int_{0}^{2 \pi}\left|p\left(e^{i \theta}\right)\right|^{2} d \theta .
$$

If $q(z)=\left(\frac{z}{k}\right)^{n} \overline{p\left(\frac{k^{2}}{z}\right)}$, then $|q(z)|=|p(z)|$ for $|z|=k$.

If $p(z) \neq 0$ in $|z|<k$ it follows that $|q(z)| \leqq|p(z)|$ for $|z|<k$. On replacing $z$ by $\frac{k^{2}}{\bar{z}}$ we deduce that for $|z|>k$, $|p(z)| \leqq|q(z)|$. Then it follows from a known result [1:p. 88 . Problem 26] that if $\mathrm{p}(\mathrm{z}) \neq 0$ in $|\mathrm{z}|<\mathrm{k}$, then for $0 \leqq \eta \leqq 2 \pi$ all the zeros of $p(z)+e^{i \eta} q(z)$ lie on the circle $|z|=k$.

Applying (5) to the polynomial $p(z)+e^{i \eta} q(z)$ we get

$$
\begin{aligned}
& \int_{0}^{2 \pi}\left|p\left(R e^{i \theta}\right)+e^{i \eta} q\left(R e^{i \theta}\right)\right|^{2} d \theta \\
& \leqq \frac{R^{2 n}+k^{2 n}}{1+k^{2 n}} \int_{0}^{2 \pi}\left|p\left(e^{i \theta}\right)+e^{i \eta} q\left(e^{i \theta}\right)\right|^{2} d \theta .
\end{aligned}
$$

We now integrate both sides with respect to $\eta$ from 0 to $2 \pi$. From the above it is clear that for $0 \leqq \theta \leqq 2 \pi$, $\left|p\left(\operatorname{Re}^{i \theta}\right)\right| \leqq\left|q\left(\operatorname{Re}^{i \theta}\right)\right|$ and $\left|p\left(e^{i \theta}\right)\right|>\left|q\left(e^{i \theta}\right)\right|$. On inverting the order of integration we obtain

$$
\begin{aligned}
& \int_{0}^{2 \pi}\left|1+e^{i \eta}\right|^{2} d \eta \times \int_{0}^{2 \pi}\left|p\left(R e^{i \theta}\right)\right|^{2} d \theta \\
& \leqq \frac{\mathrm{R}^{2 n}+k^{2 n}}{1+k^{2 n}} \int_{0}^{2 \pi}\left|p\left(e^{i \theta}\right)\right|^{2} d \theta \times \int_{0}^{2 \pi}\left|1+e^{i \eta}\right|^{2} d \eta .
\end{aligned}
$$


This proves ( 3 ).

Now $\log \int_{0}^{2 \pi}\left|p\left(r e^{i \theta}\right)\right|^{2} d \theta$ is a convex function of log $r$, and therefore for $1 \leqq \mathrm{R} \leqq \mathrm{k}^{2}$

$$
\begin{aligned}
& \left(\int_{0}^{2 \pi}\left|p\left(R e^{i \theta}\right)\right|^{2} d \theta\right) \log k^{2} \\
& \leq\left(\int_{0}^{2 \pi}\left|p\left(e^{i \theta}\right)\right|^{2} d \theta\right) \log \frac{k^{2}}{R}\left(\int_{0}^{2 \pi}\left|p\left(k^{2} e^{i \theta}\right)\right|^{2} d \theta\right) \log R \\
& \leq\left(\int_{0}^{2 \pi}\left|p\left(e^{i \theta}\right)\right|^{2} d \theta\right) \log \frac{k^{2}}{R}\left(k^{2 n}\right)^{\log R}\left(\int_{0}^{2 \pi}\left|p\left(e^{i \theta}\right)\right|^{2} d \theta\right)^{\log R} \\
& =\left(k^{2 n}\right) \log R\left(\int_{0}^{2 \pi}\left|p\left(e^{i \theta}\right)\right|^{2} d \theta\right)^{\log k^{2}}
\end{aligned}
$$

or

$$
\begin{aligned}
\left(\int_{0}^{2 \pi}\left|p\left(R e^{i \theta}\right)\right|^{2} d \theta\right) & \leqq\left(k^{2 n}\right)^{\log R / \log k^{2}}\left(\int_{0}^{2 \pi}\left|p\left(e^{i \theta}\right)\right|^{2} d \theta\right) \\
& =R^{\eta} \int_{0}^{2 \pi}\left|p\left(e^{i \theta}\right)\right|^{2} d \theta .
\end{aligned}
$$

Hence the result.

THEOREM 2. If the geometric mean of the moduli of the zeros of $\mathrm{p}(\mathrm{z})$ is at least equal to $k$, then

$$
\begin{aligned}
& \int_{0}^{2 \pi}\left|p\left(R e^{i \theta}\right)\right|^{2} d \theta \\
& \leqq\left(\frac{\left(1+k^{2 n}\right)^{\eta}+k^{2 \eta}}{1+k^{2 n}}\right)^{2 \log R / \log \left(1+k^{2 n}\right)}\left(\int_{0}^{2 \pi}\left|p\left(e^{i \theta}\right)\right|^{2} d \theta\right)
\end{aligned}
$$




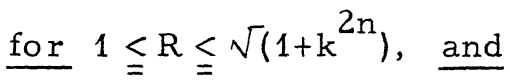

$$
\int_{0}^{2 \pi}\left|p\left(R e^{i \theta}\right)\right|^{2} d \theta \leqq \frac{R^{2 n}+k^{2 n}}{1+k^{2 n}} \int_{0}^{2 \pi}\left|p\left(e^{i \theta}\right)\right|^{2} d \theta
$$

for $R \geqq \sqrt{\left(1+k^{2 n}\right)}$.

Theorem 2 can be proved on the same lines as Theorem 2 of [2].

I am grateful to Dr. Q.I. Rahman for sugge sting this problem to me and for assisting me in the preparation of this paper.

\section{REFERENCES}

1. G. Pólya and G. Szegö: Aufgaben und Lehrsätze aus der Analysis. Vol. I. Berlin 1925.

2. Q.I. Rahman: Some Inequalities for Polynomial and Related Entire Functions. IIlinois Journal of Mathematics. Vol. 5 (1961), pp. 144-151.

Regional Engineering College

Srinagar, Kashmir, India and

Université de MontréaI

Montréal, Canada 